\title{
Several determinantal expressions of generalized Tribonacci polynomials and sequences
}

\author{
Can Kızılateş, Wei-Shih Du and Feng Qi \\ Dedicated to Dr. Professor Pietro Cerone at La Trobe University in Australia
}

\begin{abstract}
In the paper, the authors present several explicit formulas for the $(p, q, r)$ Tribonacci polynomials and generalized Tribonacci sequences in terms of the Hessenberg determinants and, consequently, derive several explicit formulas for the Tribonacci numbers and polynomials, the Tribonacci-Lucas numbers, the Perrin numbers, the Padovan (Cordonnier) numbers, the Van der Laan numbers, the Narayana numbers, the third order Jacobsthal numbers, and the third order Jacobsthal-Lucas numbers in terms of special Hessenberg determinants.
\end{abstract}

Keywords. Generalized Tribonacci sequence, Tribonacci polynomial, Tribonacci number, Hessenberg determinant

\section{Motivations}

The Tribonacci sequence $\left\{T_{n}\right\}_{n=0}^{\infty}$ and Tribonacci-Lucas sequence $\left\{K_{n}\right\}_{n=0}^{\infty}$ are defined respectively by the third order recurrence relations

$$
T_{n}=T_{n-1}+T_{n-2}+T_{n-3} \quad \text { and } \quad K_{n}=K_{n-1}+K_{n-2}+K_{n-3}
$$

for $n \geq 3$, with the initial values $T_{0}=0, T_{1}=T_{2}=1, K_{0}=3, K_{1}=1$, and $K_{2}=3$ respectively. The Tribonacci numbers $T_{n}$ were defined in [11] for the first time and some properties for $T_{n}$ have been investigated in $[6,10,11,15,17,31,34]$. In [6, Example 3.3], the determinantal expression

$$
T_{n+1}=\left|\begin{array}{ccccc}
1 & 1 & & & \\
-1 & 1 & 1 & & \\
1 & -1 & 1 & \ddots & \\
& \ddots & \ddots & \ddots & 1 \\
& & 1 & -1 & 1
\end{array}\right|_{n}, n \geq 1
$$

Received date: June 13, 2020; Published online: April 10, 2021.

2010 Mathematics Subject Classification. 11B39, 11B83, 11C20, 11Y55, 26A06, 26A09, $26 \mathrm{C} 05$. Corresponding author: Feng Qi. 
was derived. There are many generalizations of the Tribonacci sequences $T_{n}$ and $K_{n}$. One of these generalizations is the generalized Tribonacci sequence $V_{n}(x, y, z ; a, b, c)$ defined for $n \geq 3$ by

$$
\begin{aligned}
V_{n}(x, y, z ; a, b, c)=x V_{n-1}(x, y, z ; a, b, c) & \\
& +y V_{n-2}(x, y, z ; a, b, c)+z V_{n-3}(x, y, z ; a, b, c),
\end{aligned}
$$

where

$$
V_{0}(x, y, z ; a, b, c)=a, \quad V_{1}(x, y, z ; a, b, c)=b, \quad V_{2}(x, y, z ; a, b, c)=c
$$

are arbitrary integers and $x, y, z$ are real numbers. The generating function of the generalized Tribonacci sequence $V_{n}(x, y, z ; a, b, c)$ is

$$
\sum_{n=0}^{\infty} V_{n}(x, y, z ; a, b, c) t^{n}=\frac{a+(b-a x) t+(c-x b-y a) t^{2}}{1-x t-y t^{2}-z t^{3}} .
$$

There have been many studies on the generalized Tribonacci numbers $V_{n}(x, y, z ; a, b, c)$. For more information, please refer to $[3,9,30,31,33,34]$ and closely related references therein. Some special cases of the generalized Tribonacci sequence $V_{n}(x, y, z ; a, b, c)$ are as follows:

1. $V_{n}(1,1,1 ; 0,1,1)=T_{n}$, the Tribonacci sequence;

2. $V_{n}(1,1,1 ; 3,1,3)=K_{n}$, the Tribonacci-Lucas sequence;

3. $V_{n}(0,1,1 ; 3,0,2)=Q_{n}$, the Perrin sequence;

4. $V_{n}(0,1,1 ; 1,1,1)=P_{n}$, the Padovan (Cordonnier) sequence;

5. $V_{n}(0,1,1 ; 1,0,1)=R_{n}$, the Van der Laan sequence;

6. $V_{n}(1,0,1 ; 0,1,1)=N_{n}$, the Narayana sequence;

7. $V_{n}(1,1,2 ; 0,1,1)=J_{n}^{(3)}$, the third order Jacobsthal sequence;

8. $V_{n}(1,1,2 ; 2,1,5)=j_{n}^{(3)}$, the third order Jacobsthal-Lucas sequence.

In [6, Example 3.4], the determinantal expression

$$
Q_{n+1}=\left|\begin{array}{cccccc}
2 & 1 & & & & \\
-3 & 0 & 1 & & & \\
0 & -1 & 0 & 1 & & \\
& 1 & -1 & 0 & \ddots & \\
& & \ddots & \ddots & \ddots & 1 \\
& & & 1 & -1 & 0
\end{array}\right|_{n} \quad n \geq 1
$$

was derived.

In [12], the Tribonacci polynomials $T_{n}(x)$ were defined for $n \geq 3$ by

$$
T_{n}(x)=x^{2} T_{n-1}(x)+x T_{n-2}(x)+T_{n-3}(x),
$$


where $T_{0}(x)=0, T_{1}(x)=1$, and $T_{2}(x)=x^{2}$. In [6, Theorem 3.10], the determinant

$$
T_{n+1}(x)=\left|\begin{array}{ccccc}
x^{2} & 1 & & & \\
-x & x^{2} & 1 & & \\
1 & -x & x^{2} & \ddots & \\
& \ddots & \ddots & \ddots & 1 \\
& & 1 & -x & x^{2}
\end{array}\right|_{n}, \quad n \geq 1
$$

was established. In [32], the Tribonacci polynomials $T_{n}(x)$ were extended to the $(p, q, r)$-Tribonacci polynomials, denoted by $T_{p, q, r ; n}(x)$, defined for $n \geq 3$ by

$$
T_{p, q, r ; n}(x)=p(x) T_{p, q, r ; n-1}(x)+q(x) T_{p, q, r ; n-2}(x)+r(x) T_{p, q, r ; n-3}(x)
$$

with initial values $T_{p, q, r ; 0}(x)=0, T_{p, q, r ; 1}(x)=F_{p, q ; 1}(x)$, and $T_{p, q, r ; 2}(x)=F_{p, q ; 2}(x)$, where $p(x)$, $q(x), r(x)$ are non-zero polynomials with real coefficients and $F_{p, q ; n}(x)$ are the $(p, q)$-Fibonacci polynomials defined for $n \geq 2$ by

$$
F_{p, q ; n}(x)=p(x) F_{p, q ; n-1}(x)+q(x) F_{p, q ; n-2}(x)
$$

with $F_{p, q ; 0}(x)=0$ and $F_{p, q ; 1}(x)=1$. For more information on $(p, q)$-Fibonacci polynomials, please refer to [16]. The generating function of the $(p, q, r)$-Tribonacci polynomials is

$$
\sum_{n=0}^{\infty} T_{p, q, r ; n}(x) t^{n}=\frac{t}{1-p(x) t-q(x) t^{2}-r(x) t^{3}} .
$$

One of anonymous referees pointed out that the papers $[4,5,7]$ are also connected with this topic and provide the motivations of this topic.

A determinant $H=\left|h_{i j}\right|_{n \times n}$ is called a Hessenberg determinant if $h_{i j}=0$ for all pairs $(i, j)$ such that $i+1<j$ or $j+1<i$. For more information, see the papers [13, 20, 24, 29] and closely related references therein. A determinant $H=\left|h_{i j}\right|_{n \times n}$ is called a tridiagonal determinant if $h_{i j}=0$ for all pairs $(i, j)$ such that $|i-j|>1$. For more details, see [21, 25] and closely related references therein. Tridiagonal determinants are special Hessenberg determinants.

In mathematics, a closed form is a mathematical expression that can be evaluated in a finite number of operations. It may contain constants, variables, four arithmetic operations, and elementary functions, but usually no limit. It is clear that a determinant with closed elements is a closed expression. In combinatorics and number theory, representing the general term for a sequence of numbers or a sequence of polynomials as a determinant is a significant and important topic, because different closed forms demonstrate different information in mathematics. Generally, doing this is difficult.

In this paper, we will present explicit formulas for $(p, q, r)$-Tribonacci polynomials $T_{p, q, r ; n}(x)$ and for generalized Tribonacci sequences $V_{n}(x, y, z ; a, b, c)$ in terms of Hessenberg determinants and, consequently, derive explicit formulas for the Tribonacci numbers $T_{n}$, for the Tribonacci polynomials $T_{n}(x)$, for the Tribonacci-Lucas numbers $K_{n}$, for the Perrin numbers $Q_{n}$, for the Padovan (Cordonnier) numbers $P_{n}$, for the Van der Laan numbers $R_{n}$, for the Narayana numbers $N_{n}$, for the third order Jacobsthal numbers $J_{n}^{(3)}$, and for the third order Jacobsthal-Lucas numbers $j_{n}^{(3)}$ in terms of special Hessenberg determinants. By the way, those determiantal expressions for $T_{n}, Q_{n}$, and $T_{n}(x)$ derived in this paper are different from (1.1), (1.4), and (1.5). 


\section{Main results and their proofs}

Our main results can be stated as the following theorems.

Theorem 2.1. The $(p, q, r)$-Tribonacci polynomials $T_{p, q, r ; n}(x)$ for $n \geq 0$ can be represented by the special Hessenberg determinant

$$
\begin{aligned}
& T_{p, q, r ; n}(x)= \\
& \left.\qquad \begin{array}{cccccccc}
0 & -1 & 0 & 0 & \cdots & 0 & 0 & 0 \\
1 & p(x)\left(\begin{array}{l}
1 \\
0
\end{array}\right) & -1 & 0 & \cdots & 0 & 0 & 0 \\
0 & 2 q(x)\left(\begin{array}{l}
2 \\
0
\end{array}\right) & p(x)\left(\begin{array}{l}
2 \\
1
\end{array}\right) & -1 & \cdots & 0 & 0 & 0 \\
0 & 6 r(x)\left(\begin{array}{l}
3 \\
0
\end{array}\right) & 2 q(x)\left(\begin{array}{l}
3 \\
1
\end{array}\right) & p(x)\left(\begin{array}{l}
3 \\
2
\end{array}\right) & \cdots & 0 & 0 & 0 \\
0 & 0 & 6 r(x)\left(\begin{array}{l}
4 \\
1
\end{array}\right) & 2 q(x)\left(\begin{array}{l}
4 \\
2
\end{array}\right) & \cdots & 0 & 0 & 0 \\
\vdots & \vdots & \vdots & \vdots & \ddots & \vdots & \vdots & \vdots \\
0 & 0 & 0 & 0 & \cdots & -1 & 0 & 0 \\
0 & 0 & 0 & 0 & \cdots & p(x)\left(\begin{array}{c}
n-2 \\
n-3
\end{array}\right) & -1 & 0 \\
0 & 0 & 0 & 0 & \cdots & 2 q(x)\left(\begin{array}{c}
n-1 \\
n-3
\end{array}\right) & p(x)\left(\begin{array}{c}
n-1 \\
n-2
\end{array}\right) & -1 \\
n \\
n-3
\end{array}\right) \\
& 0
\end{aligned}
$$

Proof. Let $u(t)$ and $v(t) \neq 0$ be two differentiable functions, let $U_{(n+1) \times 1}(t)$ be an $(n+1) \times 1$ matrix whose elements are $u_{k, 1}(t)=u^{(k-1)}(t)$ for $1 \leq k \leq n+1$, let $V_{(n+1) \times n}(t)$ be an $(n+1) \times n$ matrix whose elements are

$$
v_{i j}(t)= \begin{cases}\left(\begin{array}{l}
i-1 \\
j-1
\end{array}\right) v^{(i-j)}(t), & i-j \geq 0 \\
0, & i-j<0\end{cases}
$$

for $1 \leq i \leq n+1$ and $1 \leq j \leq n$, and let $\left|W_{(n+1) \times(n+1)}(t)\right|$ denote the determinant of the $(n+1) \times(n+1)$ matrix

$$
W_{(n+1) \times(n+1)}(t)=\left(U_{(n+1) \times 1}(t) \quad V_{(n+1) \times n}(t)\right) .
$$

Then the $n$th derivative of the ratio $\frac{u(t)}{v(t)}$ can be computed [1, p. 40, Exercise 5] by

$$
\frac{\mathrm{d}^{n}}{\mathrm{~d} t^{n}}\left[\frac{u(t)}{v(t)}\right]=(-1)^{n} \frac{\left|W_{(n+1) \times(n+1)}(t)\right|}{v^{n+1}(t)} .
$$

Applying $u(t)=t$ and $v(t)=1-p(x) t-q(x) t^{2}-r(x) t^{3}$ in (2.2) yields

$$
\begin{aligned}
\frac{\mathrm{d}^{n}}{\mathrm{~d} t^{n}}\left[\frac{t}{1-p(x) t-q(x) t^{2}-r(x) t^{3}}\right]=\frac{(-1)^{n}}{\left(1-p(x) t-q(x) t^{2}-r(x) t^{3}\right)^{n+1}} \\
\quad \times \quad \begin{array}{ccc}
t & 1-p(x) t-q(x) t^{2}-r(x) t^{3} & \cdots \\
1 & -\left(p(x)+2 q(x) t+3 r(x) t^{2}\right)\left(\begin{array}{l}
1 \\
0
\end{array}\right) & \cdots \\
0 & -(2 q(x)+6 r(x) t)\left(\begin{array}{l}
2 \\
0
\end{array}\right) & \cdots \\
0 & -6 r(x)\left(\begin{array}{l}
3 \\
0
\end{array}\right) & \cdots \\
\vdots & \vdots & \ddots \\
0 & 0 & \cdots \\
0 & 0 & \cdots \\
0 & 0 & \cdots
\end{array}
\end{aligned}
$$




$$
\begin{aligned}
& 0 \\
& 0+0 \\
& 0 \text { 0 } \\
& 0 \text { - } \\
& 1-p(x) t-q(x) t^{2}-r(x) t^{3} \quad 0 \\
& \begin{array}{cc}
-\left(p(x)+2 q(x) t+3 r(x) t^{2}\right)\left(\begin{array}{c}
n-1 \\
n-2
\end{array}\right) & 1-p(x) t-q(x) t^{2}-r(x) t^{3} \\
-(2 q(x)+6 r(x) t)\left(\begin{array}{c}
n \\
n-2
\end{array}\right) & -\left(p(x)+2 q(x) t+3 r(x) t^{2}\right)\left(\begin{array}{c}
n \\
n-1
\end{array}\right)
\end{array} \\
& \rightarrow(-1)^{n}\left|\begin{array}{ccccc}
0 & 1 & \cdots & 0 & 0 \\
1 & -p(x)\left(\begin{array}{l}
1 \\
0
\end{array}\right) & \cdots & 0 & 0 \\
0 & -2 q(x)\left(\begin{array}{l}
2 \\
0
\end{array}\right) & \cdots & 0 & 0 \\
0 & -6 r(x)\left(\begin{array}{l}
3 \\
0
\end{array}\right) & \cdots & 0 & 0 \\
\vdots & \vdots & \ddots & \vdots & \vdots \\
0 & 0 & \cdots & 1 & 0 \\
0 & 0 & \cdots & -p(x)\left(\begin{array}{c}
n-1 \\
n-2
\end{array}\right) & 1 \\
0 & 0 & \cdots & -2 q(x)\left(\begin{array}{c}
n \\
n-2
\end{array}\right) & -p(x)\left(\begin{array}{c}
n \\
n-1
\end{array}\right)
\end{array}\right|
\end{aligned}
$$

as $t \rightarrow 0$ for $n \in \mathbb{N}$. By the generating function in (1.7), we see that

$$
\begin{aligned}
T_{p, q, r ; n}(x) & =\frac{1}{n !} \lim _{t \rightarrow 0} \frac{\mathrm{d}^{n}}{\mathrm{~d} t^{n}}\left[\begin{array}{c}
t \\
1-p(x) t-q(x) t^{2}-r(x) t^{3}
\end{array}\right] \\
& =\frac{(-1)^{n}}{n !}\left|\begin{array}{ccccc}
0 & 1 & \cdots & 0 & 0 \\
1 & -p(x)\left(\begin{array}{l}
1 \\
0
\end{array}\right) & \cdots & 0 & 0 \\
0 & -2 q(x)\left(\begin{array}{l}
2 \\
0
\end{array}\right) & \cdots & 0 & 0 \\
0 & -6 r(x)\left(\begin{array}{l}
3 \\
0
\end{array}\right) & \cdots & 0 & 0 \\
\vdots & \vdots & \ddots & \vdots & \vdots \\
0 & 0 & \cdots & 1 & 0 \\
0 & 0 & \cdots & -p(x)\left(\begin{array}{c}
n-1 \\
n-2
\end{array}\right) & 1 \\
0 & 0 & \cdots & -2 q(x)\left(\begin{array}{c}
n \\
n-2
\end{array}\right) & -p(x)\left(\begin{array}{c}
n \\
n-1
\end{array}\right)
\end{array}\right|
\end{aligned}
$$

which can be rewritten as the expression (2.1). The required proof is complete.

Theorem 2.2. The generalized Tribonacci numbers $V_{n}(x, y, z ; a, b, c)$ for $n \geq 0$ can be represented by the special Hessenberg determinant

$$
\begin{aligned}
& V_{n}(x, y, z ; a, b, c) \\
& \qquad \begin{array}{ccccccccc}
a & -1 & 0 & 0 & \cdots & 0 & 0 & 0 \\
b-a x & x\left(\begin{array}{c}
1 \\
0
\end{array}\right) & -1 & 0 & \cdots & 0 & 0 & 0 \\
2(c-b x-a y) & 2 y\left(\begin{array}{c}
2 \\
0
\end{array}\right) & x\left(\begin{array}{l}
2 \\
1
\end{array}\right) & -1 & \cdots & 0 & 0 & 0 \\
0 & 6 z\left(\begin{array}{c}
3 \\
0
\end{array}\right) & 2 y\left(\begin{array}{l}
3 \\
1
\end{array}\right) & x\left(\begin{array}{l}
3 \\
2
\end{array}\right) & \cdots & 0 & 0 & 0 \\
0 & 0 & 6 z\left(\begin{array}{l}
4 \\
1
\end{array}\right) & 2 y\left(\begin{array}{l}
4 \\
2
\end{array}\right) & \cdots & 0 & 0 & 0 \\
\vdots & \vdots & \vdots & \vdots & \ddots & \vdots & \vdots & \vdots \\
0 & 0 & 0 & 0 & \cdots & -1 & 0 & 0 \\
0 & 0 & 0 & 0 & \cdots & x\left(\begin{array}{c}
n-2 \\
n-3
\end{array}\right) & -1 & 0 \\
0 & 0 & 0 & 0 & \cdots & 2 y\left(\begin{array}{c}
n-1 \\
n-3
\end{array}\right) & x\left(\begin{array}{c}
n-1 \\
n-2
\end{array}\right) & -1 \\
0 & 0 & 0 & 0 & \cdots & 6 z\left(\begin{array}{c}
n \\
n-3
\end{array}\right) & 2 y\left(\begin{array}{c}
n \\
n-2
\end{array}\right) & x\left(\begin{array}{c}
n \\
n-1
\end{array}\right)
\end{array} \mid .
\end{aligned}
$$


Proof. Applying $u(t)=a+(b-a x) t+(c-x b-y a) t^{2}$ and $v(t)=1-x t-y t^{2}-z t^{3}$ in (2.2) gives

$$
\begin{aligned}
& \frac{\mathrm{d}^{n}}{\mathrm{~d} x^{n}}\left[\frac{a+(b-a x) t+(c-x b-y a) t^{2}}{1-x t-y t^{2}-z t^{3}}\right]=\frac{(-1)^{n}}{\left(1-x t-y t^{2}-z t^{3}\right)^{n+1}} \\
& \times \mid \begin{array}{ccc}
a+(b-a x) t+(c-x b-y a) t^{2} & 1-x t-y t^{2}-z t^{3} & \ldots \\
(b-a x)+2(c-x b-y a) t & -\left(x+2 y t+3 z t^{2}\right)\left(\begin{array}{l}
1 \\
0
\end{array}\right) & \ldots \\
2(c-x b-y a) & -(2 y+6 z t)\left(\begin{array}{l}
2 \\
0
\end{array}\right) & \cdots \\
\vdots & \vdots & \ddots \\
0 & 0 & \cdots \\
0 & 0 & \ldots \\
0 & 0 & \ldots
\end{array}
\end{aligned}
$$

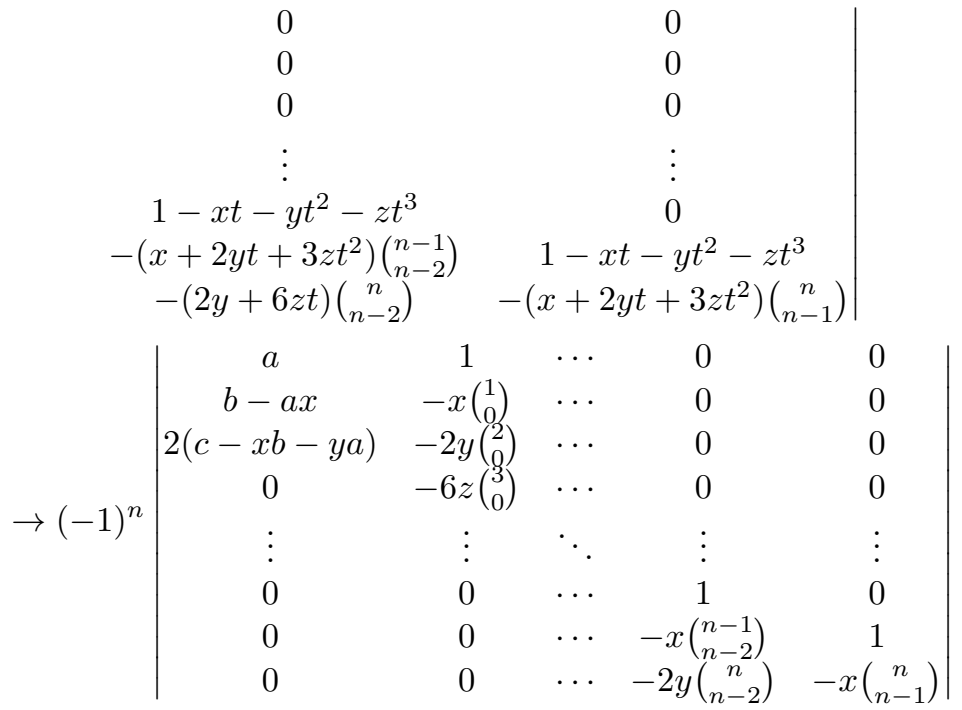

as $t \rightarrow 0$ for $n \in \mathbb{N}$. By the generating function in (1.3), we have

$$
\begin{gathered}
V_{n}(x, y, z ; a, b, c)=\frac{1}{n !} \lim _{t \rightarrow 0} \frac{\mathrm{d}^{n}}{\mathrm{~d} t^{n}}\left[\frac{a+(b-a x) t+(c-x b-y a) t^{2}}{1-x t-y t^{2}-z t^{3}}\right] \\
=\frac{(-1)^{n}}{n !}\left|\begin{array}{ccccccc}
a & 1 & 0 & 0 & \cdots & 0 & 0 \\
b-x a & -x\left(\begin{array}{l}
1 \\
0
\end{array}\right) & 1 & 0 & \cdots & 0 & 0 \\
2(c-b x-y a) & -2 y\left(\begin{array}{c}
2 \\
0
\end{array}\right) & -x\left(\begin{array}{l}
2 \\
1
\end{array}\right) & 1 & \cdots & 0 & 0 \\
0 & -6 z\left(\begin{array}{c}
3 \\
0
\end{array}\right) & -2 y\left(\begin{array}{l}
3 \\
1
\end{array}\right) & -x\left(\begin{array}{c}
3 \\
2
\end{array}\right) & \cdots & 0 & 0 \\
\vdots & \vdots & \vdots & \vdots & \ddots & \vdots & \vdots \\
0 & 0 & 0 & 0 & \cdots & 1 & 0 \\
0 & 0 & 0 & 0 & \cdots & -x\left(\begin{array}{c}
n-1 \\
n-2
\end{array}\right) & 1 \\
0 & 0 & 0 & 0 & \cdots & -2 y\left(\begin{array}{c}
n \\
n-2
\end{array}\right) & -x\left(\begin{array}{c}
n \\
n-1
\end{array}\right)
\end{array}\right|
\end{gathered}
$$

which can be rewritten as the expression (2.3).

\section{Special cases}

In this section, we will derive special cases of our main results in the form of corollaries. 
Corollary 3.1. The Tribonacci polynomials $T_{n}(x)$ for $n \geq 0$ can be represented by the special Hessenberg determinant

$$
T_{n}(x)=
$$

$$
\frac{1}{n !}\left|\begin{array}{ccccccccc}
0 & -1 & 0 & 0 & \cdots & 0 & 0 & 0 & 0 \\
1 & x^{2}\left(\begin{array}{l}
1 \\
0
\end{array}\right) & -1 & 0 & \cdots & 0 & 0 & 0 & 0 \\
0 & 2 x\left(\begin{array}{l}
2 \\
0
\end{array}\right) & x^{2}\left(\begin{array}{l}
2 \\
1
\end{array}\right) & -1 & \cdots & 0 & 0 & 0 & 0 \\
0 & 6\left(\begin{array}{c}
3 \\
0
\end{array}\right) & 2 x\left(\begin{array}{c}
3 \\
1
\end{array}\right) & x^{2}\left(\begin{array}{l}
3 \\
2
\end{array}\right) & \cdots & 0 & 0 & 0 & 0 \\
0 & 0 & 6\left(\begin{array}{l}
4 \\
1
\end{array}\right) & 2 x\left(\begin{array}{l}
4 \\
2
\end{array}\right) & \cdots & 0 & 0 & 0 & 0 \\
\vdots & \vdots & \vdots & \vdots & \ddots & \vdots & \vdots & \vdots & \vdots \\
0 & 0 & 0 & 0 & \cdots & x^{2}\left(\begin{array}{c}
n-3 \\
n-4
\end{array}\right) & -1 & 0 & 0 \\
0 & 0 & 0 & 0 & \cdots & 2 x\left(\begin{array}{c}
n-2 \\
n-4
\end{array}\right) & x^{2}\left(\begin{array}{c}
n-2 \\
n-3
\end{array}\right) & -1 & 0 \\
0 & 0 & 0 & 0 & \cdots & 6\left(\begin{array}{c}
n-1 \\
n-4
\end{array}\right) & 2 x\left(\begin{array}{c}
n-1 \\
n-3
\end{array}\right) & x^{2}\left(\begin{array}{c}
n-1 \\
n-2
\end{array}\right) & -1 \\
0 & 0 & 0 & 0 & \cdots & 0 & 6\left(\begin{array}{c}
n \\
n-3
\end{array}\right) & 2 x\left(\begin{array}{c}
n \\
n-2
\end{array}\right) & x^{2}\left(\begin{array}{c}
n \\
n-1
\end{array}\right)
\end{array}\right| .
$$

Proof. This follows from substituting $p(x)=x^{2}, q(x)=x$, and $r(x)=1$ in the equation (2.1).

Remark 1. The determiantal expressions (1.5) and (3.1) are different from each other.

Corollary 3.2. The Tribonacci numbers $T_{n}$ for $n \geq 0$ can be represented by the special Hessenberg determinant

$$
T_{n}=\frac{1}{n !}\left|\begin{array}{ccccccccc}
0 & -1 & 0 & 0 & \cdots & 0 & 0 & 0 & 0 \\
1 & \left(\begin{array}{c}
1 \\
0
\end{array}\right) & -1 & 0 & \cdots & 0 & 0 & 0 & 0 \\
0 & 2\left(\begin{array}{l}
2 \\
0
\end{array}\right) & \left(\begin{array}{c}
2 \\
1
\end{array}\right) & -1 & \cdots & 0 & 0 & 0 & 0 \\
0 & 6\left(\begin{array}{c}
3 \\
0
\end{array}\right) & 2\left(\begin{array}{l}
3 \\
1
\end{array}\right) & \left(\begin{array}{l}
3 \\
2
\end{array}\right) & \cdots & 0 & 0 & 0 & 0 \\
0 & 0 & 6\left(\begin{array}{l}
4 \\
1
\end{array}\right) & 2\left(\begin{array}{c}
4 \\
2
\end{array}\right) & \cdots & 0 & 0 & 0 & 0 \\
\vdots & \vdots & \vdots & \vdots & \ddots & \vdots & \vdots & \vdots & \vdots \\
0 & 0 & 0 & 0 & \cdots & \left(\begin{array}{c}
n-3 \\
n-4
\end{array}\right) & -1 & 0 & 0 \\
0 & 0 & 0 & 0 & \cdots & 2\left(\begin{array}{c}
n-2 \\
n-4
\end{array}\right) & \left(\begin{array}{c}
n-2 \\
n-3
\end{array}\right) & -1 & 0 \\
0 & 0 & 0 & 0 & \cdots & 6\left(\begin{array}{c}
n-1 \\
n-4
\end{array}\right) & 2\left(\begin{array}{c}
n-1 \\
n-3
\end{array}\right) & \left(\begin{array}{c}
n-1 \\
n-2
\end{array}\right) & -1 \\
0 & 0 & 0 & 0 & \cdots & 0 & 6\left(\begin{array}{c}
n \\
n-3
\end{array}\right) & 2\left(\begin{array}{c}
n \\
n-2
\end{array}\right) & \left(\begin{array}{c}
n \\
n-1
\end{array}\right)
\end{array}\right| .
$$

Proof. This follows from substituting $x=y=z=1, a=0$, and $b=c=1$ in the equation (2.3). Alternatively, the equation (3.2) can also be derived from (3.1) as $x$ tends to 1 .

Remark 2. The determiantal expressions (1.1) and (3.2) are different from each other.

Corollary 3.3. The Tribonacci-Lucas numbers $K_{n}$ for $n \geq 0$ can be represented by the special 
Hessenberg determinant

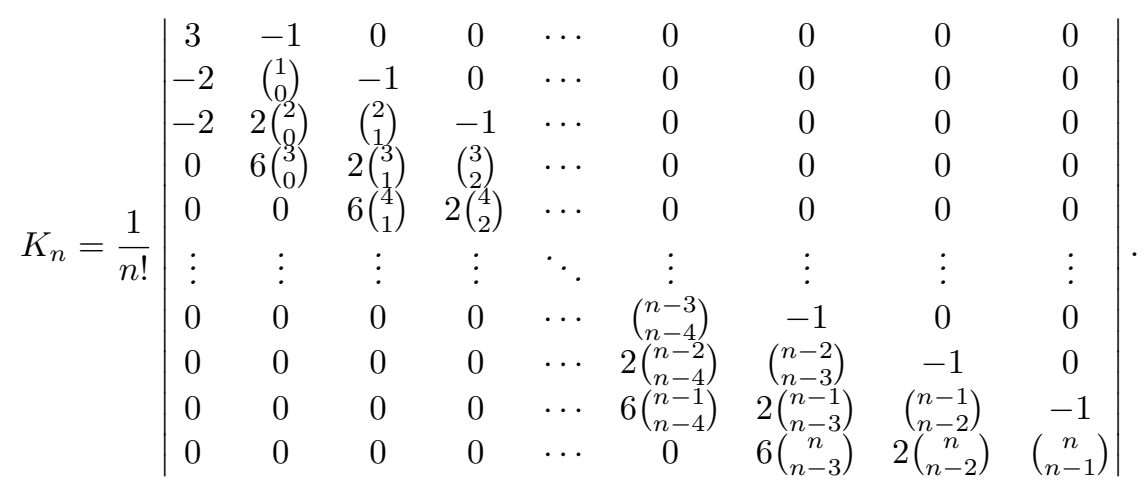

Proof. This follows from substituting $x=y=z=1, a=c=3$, and $b=1$ in the equation (2.3).

Corollary 3.4. The Perrin numbers $Q_{n}$ for $n \geq 0$ can be represented by the special Hessenberg determinant

$$
Q_{n}=\frac{1}{n !}\left|\begin{array}{ccccccccc}
3 & -1 & 0 & 0 & \cdots & 0 & 0 & 0 & 0 \\
0 & 0 & -1 & 0 & \cdots & 0 & 0 & 0 & 0 \\
-2 & 2\left(\begin{array}{c}
2 \\
0
\end{array}\right) & 0 & -1 & \cdots & 0 & 0 & 0 & 0 \\
0 & 6\left(\begin{array}{c}
3 \\
0
\end{array}\right) & 2\left(\begin{array}{c}
3 \\
1
\end{array}\right) & 0 & \cdots & 0 & 0 & 0 & 0 \\
0 & 0 & 6\left(\begin{array}{c}
4 \\
1
\end{array}\right) & 2\left(\begin{array}{c}
4 \\
2
\end{array}\right) & \cdots & 0 & 0 & 0 & 0 \\
\vdots & \vdots & \vdots & \vdots & \ddots & \vdots & \vdots & \vdots & \vdots \\
0 & 0 & 0 & 0 & \cdots & 0 & -1 & 0 & 0 \\
0 & 0 & 0 & 0 & \cdots & 2\left(\begin{array}{c}
n-2 \\
n-4
\end{array}\right) & 0 & -1 & 0 \\
0 & 0 & 0 & 0 & \cdots & 6\left(\begin{array}{c}
n-1 \\
n-4
\end{array}\right) & 2\left(\begin{array}{c}
n-1 \\
n-3
\end{array}\right) & 0 & -1 \\
0 & 0 & 0 & 0 & \cdots & 0 & 6\left(\begin{array}{c}
n \\
n-3
\end{array}\right) & 2\left(\begin{array}{c}
n \\
n-2
\end{array}\right) & 0
\end{array}\right| .
$$

Proof. This follows from substituting $x=0, y=z=1, a=3, b=0$, and $c=2$ in the equation (2.3).

Remark 3. The determinantal expressions (1.4) and (3.3) are different from each other.

Corollary 3.5. The Padovan (Cordonnier) numbers $P_{n}$ for $n \geq 0$ can be represented by the special Hessenberg determinant

$$
P_{n}=\frac{1}{n !}\left|\begin{array}{ccccccccc}
1 & -1 & 0 & 0 & \cdots & 0 & 0 & 0 & 0 \\
1 & 0 & -1 & 0 & \cdots & 0 & 0 & 0 & 0 \\
0 & 2\left(\begin{array}{c}
2 \\
0
\end{array}\right) & 0 & -1 & \cdots & 0 & 0 & 0 & 0 \\
0 & 6\left(\begin{array}{c}
3 \\
0
\end{array}\right) & 2\left(\begin{array}{c}
3 \\
1
\end{array}\right) & 0 & \cdots & 0 & 0 & 0 & 0 \\
0 & 0 & 6\left(\begin{array}{c}
4 \\
1
\end{array}\right) & 2\left(\begin{array}{c}
4 \\
2
\end{array}\right) & \cdots & 0 & 0 & 0 & 0 \\
\vdots & \vdots & \vdots & \vdots & \ddots & \vdots & \vdots & \vdots & \vdots \\
0 & 0 & 0 & 0 & \cdots & 0 & -1 & 0 & 0 \\
0 & 0 & 0 & 0 & \cdots & 2\left(\begin{array}{c}
n-2 \\
n-4
\end{array}\right) & 0 & -1 & 0 \\
0 & 0 & 0 & 0 & \cdots & 6\left(\begin{array}{c}
n-1 \\
n-4
\end{array}\right) & 2\left(\begin{array}{c}
n-1 \\
n-3
\end{array}\right) & 0 & -1 \\
0 & 0 & 0 & 0 & \cdots & 0 & 6\left(\begin{array}{c}
n \\
n-3 \\
n-3
\end{array}\right) & 2\left(\begin{array}{c}
n \\
n-2
\end{array}\right) & 0
\end{array}\right| .
$$


Proof. This follows from substituting $x=0, y=z=1$, and $a=b=c=1$ in the equation (2.3).

Corollary 3.6. The Van der Laan numbers $R_{n}$ for $n \geq 0$ can be represented by the special Hessenberg determinant

$$
R_{n}=\frac{1}{n !}\left|\begin{array}{ccccccccc}
1 & -1 & 0 & 0 & \cdots & 0 & 0 & 0 & 0 \\
0 & 0 & -1 & 0 & \cdots & 0 & 0 & 0 & 0 \\
0 & 2\left(\begin{array}{c}
2 \\
0
\end{array}\right) & 0 & -1 & \cdots & 0 & 0 & 0 & 0 \\
0 & 6\left(\begin{array}{c}
3 \\
0
\end{array}\right) & 2\left(\begin{array}{c}
3 \\
1
\end{array}\right) & 0 & \cdots & 0 & 0 & 0 & 0 \\
0 & 0 & 6\left(\begin{array}{l}
4 \\
1
\end{array}\right) & 2\left(\begin{array}{c}
4 \\
2
\end{array}\right) & \cdots & 0 & 0 & 0 & 0 \\
\vdots & \vdots & \vdots & \vdots & \ddots & \vdots & \vdots & \vdots & \vdots \\
0 & 0 & 0 & 0 & \cdots & 0 & -1 & 0 & 0 \\
0 & 0 & 0 & 0 & \cdots & 2\left(\begin{array}{c}
n-2 \\
n-4
\end{array}\right) & 0 & -1 & 0 \\
0 & 0 & 0 & 0 & \cdots & 6\left(\begin{array}{c}
n-1 \\
n-4
\end{array}\right) & 2\left(\begin{array}{c}
n-1 \\
n-3
\end{array}\right) & 0 & -1 \\
0 & 0 & 0 & 0 & \cdots & 0 & 6\left(\begin{array}{c}
n \\
n-3 \\
n-3
\end{array}\right) & 2\left(\begin{array}{c}
n \\
n-2
\end{array}\right) & 0
\end{array}\right| .
$$

Proof. This follows from substituting $x=0, y=z=1, a=c=1$, and $b=0$ in the equation (2.3).

Corollary 3.7. The Narayana numbers $N_{n}$ for $n \geq 0$ can be represented by the special Hessenberg determinant

$$
N_{n}=\frac{1}{n !}\left|\begin{array}{ccccccccc}
0 & -1 & 0 & 0 & \cdots & 0 & 0 & 0 & 0 \\
1 & \left(\begin{array}{c}
1 \\
0
\end{array}\right) & -1 & 0 & \cdots & 0 & 0 & 0 & 0 \\
0 & 0 & \left(\begin{array}{l}
2 \\
1
\end{array}\right) & -1 & \cdots & 0 & 0 & 0 & 0 \\
0 & 6\left(\begin{array}{c}
3 \\
0
\end{array}\right) & 0 & \left(\begin{array}{c}
3 \\
2
\end{array}\right) & \cdots & 0 & 0 & 0 & 0 \\
0 & 0 & 6\left(\begin{array}{c}
4 \\
1
\end{array}\right) & 0 & \cdots & 0 & 0 & 0 & 0 \\
\vdots & \vdots & \vdots & \vdots & \ddots & \vdots & \vdots & \vdots & \vdots \\
0 & 0 & 0 & 0 & \cdots & \left(\begin{array}{c}
n-3 \\
n-4
\end{array}\right) & -1 & 0 & 0 \\
0 & 0 & 0 & 0 & \cdots & 0 & \left(\begin{array}{c}
n-2 \\
n-3
\end{array}\right) & -1 & 0 \\
0 & 0 & 0 & 0 & \cdots & 6\left(\begin{array}{c}
n-1 \\
n-4
\end{array}\right) & 0 & \left(\begin{array}{c}
n-1 \\
n-2
\end{array}\right) & -1 \\
0 & 0 & 0 & 0 & \cdots & 0 & 6\left(\begin{array}{c}
n \\
n-3
\end{array}\right) & 0 & \left(\begin{array}{c}
n \\
n-1
\end{array}\right)
\end{array}\right| .
$$

Proof. This follows from substituting $x=z=1, y=0, a=0$, and $b=c=1$ in the equation (2.3).

Corollary 3.8. The third order Jacobsthal numbers $J_{n}^{(3)}$ for $n \geq 0$ can be represented by the 
special Hessenberg determinant

$$
J_{n}^{(3)}=\frac{1}{n !}\left|\begin{array}{ccccccccc}
0 & -1 & 0 & 0 & \cdots & 0 & 0 & 0 & 0 \\
1 & \left(\begin{array}{c}
1 \\
0
\end{array}\right) & -1 & 0 & \cdots & 0 & 0 & 0 & 0 \\
0 & 2\left(\begin{array}{l}
2 \\
0
\end{array}\right) & \left(\begin{array}{l}
2 \\
1
\end{array}\right) & -1 & \cdots & 0 & 0 & 0 & 0 \\
0 & 12\left(\begin{array}{c}
3 \\
0
\end{array}\right) & 2\left(\begin{array}{l}
3 \\
1
\end{array}\right) & \left(\begin{array}{l}
3 \\
2
\end{array}\right) & \cdots & 0 & 0 & 0 & 0 \\
0 & 0 & 12\left(\begin{array}{l}
4 \\
1
\end{array}\right) & 2\left(\begin{array}{c}
4 \\
2
\end{array}\right) & \cdots & 0 & 0 & 0 & 0 \\
\vdots & \vdots & \vdots & \vdots & \ddots & \vdots & \vdots & \vdots & \vdots \\
0 & 0 & 0 & 0 & \cdots & \left(\begin{array}{c}
n-3 \\
n-4
\end{array}\right) & -1 & 0 & 0 \\
0 & 0 & 0 & 0 & \cdots & 2\left(\begin{array}{c}
n-2 \\
n-4
\end{array}\right) & \left(\begin{array}{c}
n-2 \\
n-3
\end{array}\right) & -1 & 0 \\
0 & 0 & 0 & 0 & \cdots & 12\left(\begin{array}{c}
n-1 \\
n-4
\end{array}\right) & 2\left(\begin{array}{c}
n-1 \\
n-3
\end{array}\right) & \left(\begin{array}{c}
n-1 \\
n-2
\end{array}\right) & -1 \\
0 & 0 & 0 & 0 & \cdots & 0 & 12\left(\begin{array}{c}
n \\
n \\
n-3
\end{array}\right) & 2\left(\begin{array}{c}
n \\
n-2 \\
n-2
\end{array}\right) & \left(\begin{array}{c}
n \\
n-1
\end{array}\right)
\end{array}\right| .
$$

Proof. This follows from substituting $x=y=1, z=2, a=0$, and $b=c=1$ in the equation (2.3).

Corollary 3.9. The third order Jacobsthal-Lucas numbers $j_{n}^{(3)}$ for $n \geq 0$ can be represented by the special Hessenberg determinant

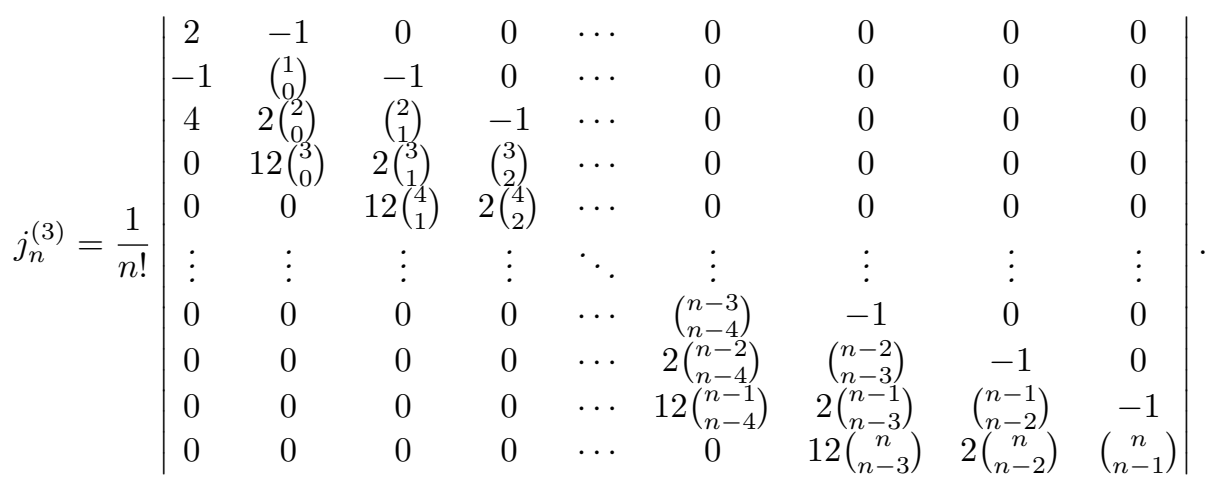

Proof. This follows from substituting $x=y=1, z=2, a=2, b=1$, and $c=5$ in the equation (2.3).

\section{Some comments}

Our authors have written down several explicit determinants which give the Tribonacci numbers (or more generally, generalized tribonacci polynomials). Determinantal expressions are well known for sequences like the Fibonacci and Tribonacci sequences defined by linear recursions using their companion matrices and raising the matrix to an appropriate power, see [2, 6, 21]. On the first glance, the expressions in this paper seem like variations of these well known expressions, but, after comparing them for a while, it is sure that they are not the same. Firstly, the matrices are not tridiagonal. For tridiagonal matrices, the $n$th determinant is expressible as a linear combination of the previous two determinants. Therefore, tridiagonal determinants are easy to be computed. In this sense, the determinantal expressions in this paper are new and different from the known ones.

The proof of the main result is really easy and is an immediate consequence of the formula (2.2) appeared in [1], which expresses the $n$th derivative of the rational function (1.7) as a 
determinant. Our authors use the rational generating function (1.7) to find the Taylor coefficient using this determinant. Using the formula (2.2), the proof of the main theorem is almost immediate and very elementary. Although most of the contents of this paper are the special cases or consequences of Theorems 2.1 and 2.2, but these expressions are new.

More generally, one can consider the generating function $\frac{1}{1-p_{1}(x) t-p_{2}(x) t^{2}-\cdots-p_{d}(x) t^{d}}$ and easily and elementarily compute its $n$th derivative by the formula (2.2).

Let $D_{0}=1$ and

$$
D_{n}=\left|\begin{array}{cccccc}
e_{1,1} & e_{1,2} & 0 & \ldots & 0 & 0 \\
e_{2,1} & e_{2,2} & e_{2,3} & \ldots & 0 & 0 \\
e_{3,1} & e_{3,2} & e_{3,3} & \ldots & 0 & 0 \\
\vdots & \vdots & \vdots & \vdots & \vdots & \vdots \\
e_{n-2,1} & e_{n-2,2} & e_{n-2,3} & \ldots & e_{n-2, n-1} & 0 \\
e_{n-1,1} & e_{n-1,2} & e_{n-1,3} & \ldots & e_{n-1, n-1} & e_{n-1, n} \\
e_{n, 1} & e_{n, 2} & e_{n, 3} & \ldots & e_{n, n-1} & e_{n, n}
\end{array}\right|
$$

for $n \in \mathbb{N}$. In [2, p. 222, Theorem], it was proved that the sequence $D_{n}$ for $n \geq 0$ satisfies $D_{1}=e_{1,1}$ and

$$
D_{n}=\sum_{s=1}^{n}(-1)^{n-s} e_{n, s}\left(\prod_{j=s}^{n-1} e_{j, j+1}\right) D_{s-1}
$$

for $n \geq 2$, where the empty product is understood to be 1. See also [23, Lemma 5], [24, Lemma 2], [28, Lemma 2], and [29, Remark 3].

Applying $D_{n}$ in (4.1) to the determinant $(n-1) ! T_{p, q, r ; n-1}(x)$ for $n \geq 1$ in (2.1) and simplifying lead to the recursion (1.6).

Applying $D_{n}$ in (4.1) to the determinant $(n-1) ! V_{n-1}(x, y, z ; a, b, c)$ for $n \geq 1$ in $(2.3)$ and simplifying result in the recursion (1.2).

We think that one of the real aims of mathematics is to simply, concisely, straightforwardly, immediately, standardly, intrinsicly, concretely, and beautifully solve difficult, complicated, complex, abstract, confused, general, and applicable problems.

The third author of this paper knew the formula (2.2), a general formula of any higher order derivative for a ratio of two differentiable functions on 25 September 2014. As acknowledged in [19, Acknowledgments] and [27, Acknowledgments], we here thank again Sergei M. Sitnik at Voronezh Institute of the Ministry of Internal Affairs of Russia for his providing the formula (2.2) and the reference [1] on 25 September 2014. Till now it seems that the general and fundamental formula (2.2) has not been extensively known and not considerably used in mathematical community.

Using the formula (2.2) together with (4.1), one can simply, elementarily, easily, standardly, and immediately obtain determinantal expressions in terms of some Hessenberg determinants and can simply, elementarily, easily, standardly, and immediately derive recursive relations of coefficients in power series expansions of functions in the form of a ratio of two infinitely differentiable functions. We believe that this approach should be useful and applicable in analytic combinatorics, analytic number theory, the theory of matrices, the theory of special functions, and other mathematical branches, as done in $[8,18,20,22,23,24,25,26,28,29]$ and closely related references therein. 


\section{Conclusions}

The formula (2.2) is a direct and effectual tool to compute and express higher order derivatives of a ratio of two differentiable functions in terms of the Hessenberg determinants. In this paper, utilizing the formula (2.2) and considering the generating functions (1.3) and (1.7) as ratios, we present explicit formulas for $(p, q, r)$-Tribonacci polynomials $T_{p, q, r ; n}(x)$ and generalized Tribonacci sequences $V_{n}(x, y, z ; a, b, c)$ in terms of special Hessenberg determinants, derive explicit formulas for the Tribonacci polynomials $T_{n}(x)$, the Tribonacci numbers $T_{n}$, the Tribonacci-Lucas numbers $K_{n}$, the Perrin numbers $Q_{n}$, the Padovan (Cordonnier) numbers $P_{n}$, the Van der Laan numbers $R_{n}$, the Narayana numbers $N_{n}$, the third order Jacobsthal numbers $J_{n}^{(3)}$, and the third order Jacobsthal-Lucas numbers $j_{n}^{(3)}$ in terms of special Hessenberg determinants.

\section{Funding}

The second author was supported by the Ministry of Science and Technology of the Republic of China (Grant No. MOST 109-2115-M-017-002-).

\section{Acknowledgments}

The authors appreciate anonymous referees for their careful corrections, helpful suggestions, and valuable comments on the original version of this paper.

Remark 4. This paper is a slightly revised version of the electronic preprint [14].

\section{References}

[1] N. Bourbaki, Functions of a Real Variable, Elementary Theory, Translated from the 1976 French original by Philip Spain, Elements of Mathematics (Berlin), Springer-Verlag, Berlin, 2004; available online at https://doi.org/10.1007/978-3-642-59315-4.

[2] N. D. Cahill, J. R. D'Errico, D. A. Narayan, and J. Y. Narayan, Fibonacci determinants, College Math. J. 33 (2002), No. 3, 221-225; available online at http://dx.doi.org/10. $2307 / 1559033$.

[3] G. Cerda-Morales, On the third-order Jacobsthal and third-order Jacobsthal-Lucas sequences and their matrix representations, arXiv preprint (2018), available online at https://arxiv . org/abs/1806.03709.

[4] G. Cerda-Morales, Some results on dual third-order Jacobsthal quaternions, Filomat 33 (2019), No. 7, 1865-1876; available online at https://doi.org/10.2298/fil1907865c.

[5] G. Cerda-Morales, Third-order Jacobsthal generalized quaternions, J. Geom. Symmetry Phys. 50 (2018), 11-27; available online at https://doi.org/10.7546/ jgsp-50-2018-11-27.

[6] J. L. Cereceda, Determinantal representations for generalized Fibonacci and Tribonacci numbers, Int. J. Contemp. Math. Sci. 9 (2014), No. 6, 269-285; available online at http://dx.doi.org/10.12988/ijcms.2014.4323. 
[7] C. K. Cook and M. R. Bacon, Some identities for Jacobsthal and JacobsthalLucas numbers satisfying higher order recurrence relations, Ann. Math. Inform. 41 (2013), 27-39.

[8] M. C. Dağlı and F. Qi, Several closed and determinantal forms for convolved Fibonacci numbers, J. Appl. Math. Stat. Inform., 17(2021), No. 1; available online at https://doi. org/10.31219/osf .io/e25yb.

[9] T. V. Didkivska and M. V. Stopochkina, Properties of Fibonacci-Narayana numbers, World Math. 9 (2003), No. 1, 29-36.

[10] M. Elia, Derived sequences, the Tribonacci recurrence and cubic forms, Fibonacci Quart. 39 (2001), No. 2, 107-115.

[11] M. Feinberg, Fibonacci-Tribonacci, Fibonacci Quart. 1 (1963), No. 3, 70-74.

[12] V. E. Hoggatt and M. Bicknell, Generalized Fibonacci polynomials, Fibonacci Quart. 11 (1973), No. 5, 457-465.

[13] S. Hu and M.-S. Kim, Two closed forms for the Apostol-Bernoulli polynomials, Ramanujan J. 46 (2018), No. 1, 103-117; available online at https://doi.org/10.1007/ s11139-017-9907-4.

[14] C. Kızılateş, W.-S. Du, and F. Qi, Several determinantal expressions of generalized Tribonacci polynomials and sequences, Authorea Preprints (2020), available online at https: //doi.org/10.22541/au.159474102.22328885.

[15] T. Koshy, Fibonacci and Lucas Numbers with Applications, Vol. 1, Second edition, Pure and Applied Mathematics (Hoboken), John Wiley \& Sons, Inc., Hoboken, NJ, 2018.

[16] G. Y. Lee and M. Asci, Some properties of the $(p, q)$-Fibonacci and $(p, q)$-Lucas polynomials, J. Appl. Math. 2012. Article ID 264842; 18 pages; available online at https://doi.org/ $10.1155 / 2012 / 264842$.

[17] S. Pethe, Some identities for Tribonacci sequences, Fibonacci Quart. 26 (1988), No. 2, $144-151$.

[18] F. Qi, A determinantal expression and a recursive relation of the Delannoy numbers, Acta Univ. Sapientiae Math. 13 (2021), No. 1, in press; available online at https://doi.org/ 10.2478/ausm-2021-????.

[19] F. Qi, Derivatives of tangent function and tangent numbers, Appl. Math. Comput. 268 (2015), 844-858; available online at http://dx.doi.org/10.1016/j .amc.2015.06.123.

[20] F. Qi, Determinantal expressions and recurrence relations for Fubini and Eulerian polynomials, J. Interdiscip. Math. 22 (2019), No. 3, 317-335; available online at https: //doi.org/10.1080/09720502.2019.1624063.

[21] F. Qi, V. Čerňanová, and Y. S. Semenov, Some tridiagonal determinants related to central Delannoy numbers, the Chebyshev polynomials, and the Fibonacci polynomials, Politehn. Univ. Bucharest Sci. Bull. Ser. A Appl. Math. Phys. 81 (2019), No. 1, 123-136.

[22] F. Qi, M. C. Dağlı, and W.-S. Du, Determinantal forms and recursive relations of the Delannoy two-functional sequence, Adv. Theory Nonlinear Anal. Appl. 4 (2020), No. 3, 184-193; available online at https://doi.org/10.31197/atnaa.772734. 
[23] F. Qi and B.-N. Guo, A diagonal recurrence relation for the Stirling numbers of the first kind, Appl. Anal. Discrete Math. 12 (2018), No. 1, 153-165; available online at https: //doi.org/10.2298/AADM170405004Q.

[24] F. Qi and B.-N. Guo, Some determinantal expressions and recurrence relations of the Bernoulli polynomials, Mathematics 4 (2016), No. 4, Article 65, 11 pages; available online at https://doi.org/10.3390/math4040065.

[25] F. Qi, C. Kızılates, and W.-S. Du, A closed formula for the Horadam polynomials in terms of a tridiagonal determinant, Symmetry 11 (2019), No. 6, 8 pages; available online at http: //dx.doi.org/10.3390/sym11060000.

[26] F. Qi, O. Kouba, and I. Kaddoura, Computation of several Hessenberg determinants, Math. Slovaca 70 (2020), No. 6, 1521-1537; available online at https://doi.org/10.1515/ $\mathrm{ms}-2017-0445$,

[27] F. Qi, X.-T. Shi, and F.-F. Liu, Several identities involving the falling and rising factorials and the Cauchy, Lah, and Stirling numbers, Acta Univ. Sapientiae Math. 8 (2016), No. 2, 282-297; available online at https://doi.org/10.1515/ausm-2016-0019.

[28] F. Qi and J.-L. Zhao, Some properties of the Bernoulli numbers of the second kind and their generating function, Bull. Korean Math. Soc. 55 (2018), No. 6, 1909-1920; available online at https://doi.org/10.4134/BKMS.b180039.

[29] F. Qi, J.-L. Zhao, and B.-N. Guo, Closed forms for derangement numbers in terms of the Hessenberg determinants, Rev. R. Acad. Cienc. Exactas Fís. Nat. Ser. A Mat. RACSAM 112 (2018), No. 4, 933-944; available online at https://doi.org/10.1007/ s13398-017-0401-z.

[30] A. G. Shannon, P. G. Anderson, and A. F. Horadam, Properties of Cordonnier, Perrin and Van der Laan numbers, Internat. J. Math. Ed. Sci. Tech. 37 (2006), No. 7, 825-831; available online at https://doi.org/10.1080/00207390600712554.

[31] A. G. Shannon and A. F. Horadam, Some properties of third-order recurrence relations, Fibonacci Quart. 10 (1972), No. 2, 135-145.

[32] M. Shattuck and E. Tan, Incomplete generalized ( $p, q, r)$-Tribonacci polynomials, Appl. Appl. Math. 13 (2018), No. 1, 1-18.

[33] M. Tan and Y. Zhang, A note on bivariate and trivariate Fibonacci polynomials, Southeast Asian Bull. Math. 29 (2005), No. 5, 975-990.

[34] C. C. Yalavigi, Properties of Tribonacci numbers, Fibonacci Quart. 10 (1972), No. 3, 231-246.

Can Kızılateş Department of Mathematics, Faculty of Arts and Sciences, Zonguldak Bülent Ecevit University, Zonguldak, Turkey

E-mail: cankizilates@gmail.com

Wei-Shih Du Department of Mathematics, National Kaohsiung Normal University, Kaohsiung 82444, Taiwan 
E-mail: wsdu@mail.nknu.edu.tw

Feng Qi School of Mathematical Sciences, Tianjin Polytechnic University, Tianjin 300387, China

E-mail: qifeng618@gmail.com 